\title{
Aneurysmal subarachnoid hemorrhage: factors associated with worse functional outcome
}

\author{
L Sánchez Montori", J González Robledo, ME Pérez Losada, M Pérez Cheng, A Rodríguez Encinas \\ From ESICM LIVES 2015 \\ Berlin, Germany. 3-7 October 2015
}

\section{Introduction}

Subarachnoid hemorrhage (SAH) is a devastating neurological emergency. Although it is a relatively common and potentially curable disease, it presents a high morbidity and mortality.

\section{Objectives}

Describe the factors associated with worse functional outcome, determined by an Extended Glasgow Outcame Scale (GOSE) of 2-3, in patients with a spontaneous subarachnoid hemorrhage of aneurysmal origin in our center.

\section{Methods}

Data from patients admitted to the ICU with SAH between January 2006 and December 2014 were retrospectively collected. We analyzed epidemiological variables, past history, severity scale scores (Glasgow Coma Scale at admission, Hunt-Hess, World Federation of Neurosurgical Societies Scale, Fisher and APACHE II), diagnostic methods, therapeutic management, complications, functional status at discharge and mortality. T test was used to compare means and logistic regression for assessing predictors. Data was analyzed with SPSS v19.

\section{Results}

We included 151 patients, with a mortality rate of $17.2 \%$. GOSE $2-3$ was found in $28.8 \%$ and $4-5$ in $71.2 \%$. Patients with worse outcome were older [64.47 (SD 12.64) vs 56.92 (SD 12.93)], with higher APACHE II [20.42 (SD 6.85) vs 13.15 (SD 6.60)], worst GCS at admission [8 (SD 4.03) vs 13.2 (SD 3.01)], higher scores on the prognostic scales: Hunt-Hess IV-V (75\% vS $16.8 \%$ ), Fischer IV ( $91.7 \%$ vs $57.3 \%$ ), WFNS IV-V $80.5 \%$ vs $25.9 \%$ ). These differences were not statistically significant, except for the GCS at admission. Complications were more frequent in
Table 1 Logistic regression analysis.

\begin{tabular}{llll}
\hline VARIABLE & OR & Cl 95\% OR & $\mathbf{p}$ \\
\hline Rebleeding & 5.13 & $1.40-18.80$ & 0,012 \\
\hline Hidrocephalus & 9.58 & $3.94-23.29$ & $<0.001$ \\
\hline Ventriculitis & 2.56 & $0.35-18.90$ & 0.365 \\
\hline Intracraneal hypertension & 7.46 & $2.68-20.72$ & $<0.001$ \\
\hline Delayed ischemic deficit & 1.27 & $0.40-4.03$ & 0.68 \\
\hline GCS 3-8 & 20.47 & $7.04-59.52$ & $<0.001$ \\
\hline GCS 9-13 & 3.87 & $1.15-13.01$ & $<0.001$ \\
\hline
\end{tabular}

the GOSE $2-3$ group: hydrocephalus (72.2\% vs $21.3 \%)$, intracranial hypertension ( $38.9 \%$ vs $7.9 \%$ ) and rebleeding $(19.4 \%$ vs $4.5 \%)$ were statistically significant. Logistic regression analysis results are summarized at table 1 :

\section{Conclusions}

SAH has a high morbidity and mortality. In our series, factors associated with worse functional outcome at discharge were GCS $\leq 13$ at admission, presence of hydrocephalus, intracranial hypertension and rebleeding.

\section{Published: 1 October 2015}

\section{References}

1. Steiner T, Juvela S, Unterberg A, Jung C, Forsting M, Rinkel G: European Stroke Organization Guidelines for the Management of Intracranial Aneurysms and Subarachnoid Haemorrhage. Cerebrovascular Diseases 2013, 35:93-112, February 7.

2. Michael N, Diringer, Thomas P, Bleck J, Claude Hemphill III, David Menon Lori Shutter, Paul Vespa, Nicolas Bruder E, Sander Connolly Jr, Giuseppe Citerio, Daryl Gress, Daniel Hänggi, Brian L. Hoh, Giuseppe Lanzino Peter Le Roux, Alejandro Rabinstein , Erich Schmutzhard, Nino Stocchetti , Jose I. Suarez, Miriam Treggiari, Ming-Yuan Tseng, Mervyn DI, Vergouwen, Stefan Wolf, Gregory Zipfel : Critical Care Management of Patients Following Aneurysmal Subarachnoid Hemorrhage: Recommendations from the Neurocritical Care Society's Multidisciplinary Consensus Conference. 
doi:10.1186/2197-425X-3-S1-A782

Cite this article as: Sánchez Montori et al: Aneurysmal subarachnoid

hemorrhage: factors associated with worse functional outcome. Intensive

Care Medicine Experimental 2015 3(Suppl 1):A782.

Submit your manuscript to a SpringerOpen ${ }^{\circ}$ journal and benefit from:

- Convenient online submission

- Rigorous peer review

- Immediate publication on acceptance

- Open access: articles freely available online

- High visibility within the field

- Retaining the copyright to your article

Submit your next manuscript at $\gg$ springeropen.com 